\title{
Physical activity, black carbon exposure and airway inflammation in an urban adolescent cohort
}

\author{
Stephanie. Lovinsky-Desir ${ }^{\mathrm{a}, *}$, Kyung Hwa Jung ${ }^{\mathrm{b}}$, Andrew G. Rundle ${ }^{\mathrm{c}}$, Lori A. Hoepner ${ }^{\mathrm{d}, \mathrm{e}}$, \\ Joshua B. Bautista ${ }^{\mathrm{b}}$, Frederica P. Perera ${ }^{\mathrm{d}}$, Steven N. Chillrud ${ }^{\mathrm{f}}$, Matthew S. Perzanowski ${ }^{\mathrm{d}}$, \\ Rachel L. Miller ${ }^{\mathrm{b}, \mathrm{d}, \mathrm{g}}$ \\ a Division of Pediatric Pulmonology, Department of Pediatrics, College of Physicians and Surgeons, Columbia University, 3959 Broadway CHC-745, New York, \\ NY 10032, United States \\ b Division of Pulmonary, Allergy and Critical Care of Medicine, Department of Medicine, College of Physicians and Surgeons, Columbia University, PH8E-101, \\ 630 W. 168S., New York, NY 10032, United States \\ ${ }^{c}$ Department of Epidemiology, Mailman School of Public Health, Columbia University, 722 W. 168S., New York, NY 10032, United States \\ ${ }^{\mathrm{d}}$ Department of Environmental Health Sciences, Mailman School of Public Health, Columbia University, 722 W. 168S., New York, NY 10032, United States \\ e Department of Environmental and Occupational Health Sciences, State University of New York, Downstate School of Public Health, Box 43, 450 Clarkson \\ Avenue, Brooklyn, NY 11203, United States \\ ${ }^{\mathrm{f}}$ Lamont-Doherty Earth Observatory, Columbia University, 61 Rt, 9W Palisades, New York 10964, United States \\ ${ }^{\mathrm{g}}$ Division of Pediatric Allergy, Immunology, and Rheumatology, Department of Pediatrics, College of Physicians and Surgeons, Columbia University, PH8E-101, \\ 630 W. 168 St., New York, NY 10032, United States
}

\section{A R T I C L E I N F O}

\section{Article history:}

Received 7 July 2016

Received in revised form

7 September 2016

Accepted 8 September 2016

\section{Keywords:}

FeNO

Exercise

Air pollution

Urban environment

Asthma

\begin{abstract}
A B S T R A C T
Objective: Regular physical activity can improve cardiopulmonary health; however, increased respiratory rates and tidal volumes during activity may increase the effective internal dose of air pollution exposure. Our objective was to investigate the impact of black carbon (BC) measured by personal sampler on the relationship between physical activity and fractional exhaled nitric oxide (FeNO), a marker of airway inflammation. We hypothesized that higher personal $\mathrm{BC}$ would attenuate the protective effect of physical activity on airway inflammation.

Methods: We performed a cross-sectional study nested in a birth cohort of African American and Dominican children living in the Bronx and Northern Manhattan, New York City. Children were recruited based on age (target 9-14 year olds) and presence $(n=70)$ or absence $(n=59)$ of current asthma. Children wore wrist mounted accelerometers for 6 days and were classified as 'active' if they had $\geq 60$ min of moderate-to-vigorous activity (MVA) each day and 'non-active' if they had $<60$ min of MVA on any given day, based on CDC guidelines. Personal BC measured using a MicroAeth, was assessed during two 24-h periods, at the beginning and end of physical activity assessment. High $\mathrm{BC}$ was defined as the upper tertile of $\mathrm{BC}$ measured with personal sampler. FeNO measurements were sampled at the beginning and end of the of physical activity assessment. Results: In multivariable linear regression models, 'active' children had $25 \%$ higher personal BC concentrations $(p=0.02)$ and $20 \%$ lower FeNO $(p=0.04)$ compared to 'non-active' children. Among children with high personal BC $(n=33)$, there was no relationship between activity and FeNO $(p=1.00)$. The significant protective relationship between activity and airway inflammation was largely driven by children with lower personal $\mathrm{BC}$ $(\mathrm{n}=96, \mathrm{p}=0.04)$.

Conclusions: Children that live in an urban environment and are physically active on a daily basis have higher personal exposure to BC. High BC offsets the protective relationship between physical activity and airway inflammation.
\end{abstract}

(c) 2016 Elsevier Inc. All rights reserved.

\footnotetext{
Abbreviations: BC, black carbon; BMI, body mass index; CCCEH, Columbia Center for Children's Environmental Health; CDC, Centers for Disease Control and Prevention; EC, elemental carbon; FeNO, fractional exhaled nitric oxide; GM, geometric mean; Gx2, mixed grass pollen; IgE, Immunoglobulin E; MVA, moderate-tovigorous activity; $\mathrm{NO}$, nitric oxide; $\mathrm{NO}_{2}$, nitrogen dioxide; NYC, New York City; PAH, polycyclic aromatic hydrocarbon; PM, particulate matter; SD, standard deviation; TRAP, traffic related air pollution; Tx1, mixed tree pollen; VA, vigorous activity

* Corresponding author.

E-mail address: sl3230@cumc.columbia.edu (Stephanie. Lovinsky-Desir).
}

\section{Introduction}

Physical activity provides numerous health benefits, including improved metabolic and cardiopulmonary health (Strong et al., 2005). The Centers for Disease Control and Prevention (CDC) recommend that children participate in at least $60 \mathrm{~min}$ of moderate or vigorous physical activity daily with at least 3 days a week of 
vigorous activity (Strong et al., 2005). However, increased respiratory rates and tidal volumes during physical activity may increase the effective internal dose of air pollution exposure (Oravisjarvi et al., 2011). This could be especially critical in urban environments where options for physical activity may occur near sources of higher traffic-related air pollution (TRAP) due to the structure of the built environment (Council on Sports et al., 2006). However, very little research to date has addressed the link in children between regular physical activity and pollutant exposures in urban environments.

Exposure to TRAP is associated with wheeze, cough, respiratory symptoms (Spira-Cohen et al., 2011) and childhood asthma (McConnell et al., 2006). Of particular interest is, black carbon (BC), a major indicator of TRAP exposure in urban cities and a contributor to global warming (Wallack and Ramanathan, 2009) that results from incomplete combustion of fossil fuels, biofuels, and biomass. BC exposure has been associated with increased airway inflammation in children living in urban environments (Cornell et al., 2012; Lin et al., 2011). In contrast, the well-established biomarker of allergic airway inflammation, fractional exhaled nitric oxide (FeNO) (Dweik et al., 2011), has been shown to be reduced in response to physical activity (Mendes et al., 2011). Thus, it is important to better understand the balance between the benefits of regular physical activity in children the potential harm from exposure to ambient pollutants in urban environments.

Our objective was to determine the impact of personal exposure to the pollutant $\mathrm{BC}$ on the relationship between physical activity and airway inflammation. First, we hypothesized that active children that live in an urban environment would have greater exposure to BC, measured with personal sampler. Second, we hypothesized that higher personal $\mathrm{BC}$ exposure may reduce the magnitude of protection induced by physical activity on airway inflammation. Our approach was to investigate children ages 9-14 years that live in Northern Manhattan and the Bronx, NYC in a nested study that is part of a longitudinal birth cohort.

\section{Materials and methods}

\subsection{Study population}

Study participants $(n=163)$ were recruited from the Columbia Center for Children's Environmental Health (CCCEH) longitudinal birth cohort comprised of children living in Northern Manhattan and the South Bronx of NYC, whose non-smoking, African American and Dominican mothers were enrolled during pregnancy (Perera et al., 2003). As part of the research design for the nested cross-sectional 'parent study', participants were recruited based on age (9-14 year olds) and current asthma diagnosis (LovinskyDesir et al., 2014). Asthma was determined by physician diagnosis during at least one cohort study visit between ages 5-12 years (Donohue et al., 2013) and report of asthma symptoms or asthma medication use in the 12 months prior to enrollment.

Children with body mass index (BMI) $\geq$ the 95th percentile for age and sex were classified as 'obese'. Children were recruited across all 4 meteorological seasons. Complete data on physical activity, personal BC measures, FeNO and allergic sensitization (an important covariate for examination of FeNO) was available for $\mathrm{n}=129$ children that were included in this study (Fig. 1). The longitudinal birth cohort study is conducted in accordance with Columbia University Institutional Review Board guidelines and informed consents and assents were obtained.

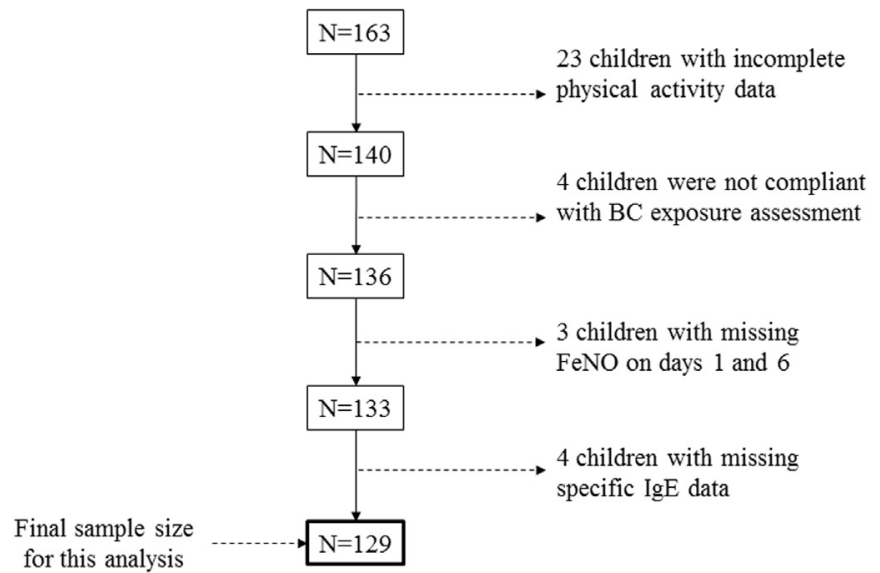

Fig. 1. Schematic representation of sample size. Of the 163 children enrolled in the parent study, 23 children had incomplete physical activity data, 4 children did not meet compliance standards for wearing personal BC exposure equipment, 3 children had missing FeNO measurements on both sampling days and 4 children did not have specific IgE data yielding a final sample size of 129 children for the current analysis. BC: black carbon, FeNO: fractional exhaled nitric oxide, IgE: Immunoglobulin E.

\subsection{Physical activity assessment}

All study participants wore an accelerometer (Actical, Philips Respironics, Bend, OR) continuously on the non-dominant wrist for 6 consecutive days (Fig. 2) and integrated measures of gross motor activity were measured in 1-min epochs. Data collection began and ended on a weekday in order to capture both weekday and weekend activity. To account for having collected only partial activity data on the first and last days we used only the 5 intervening consecutive days with full 24-h of data for characterization of physical activity (Rundle et al., 2009).

Physical activity was characterized based on the CDC recommendation that children participate in $60 \mathrm{~min}$ or more of moderate-to-vigorous physical activity (MVA = moderate + vigorous activity) each day and vigorous activity (VA) on at least 3 days per week (http://www.cdc.gov/physicalactivity/everyone/guidelines/children. html). Using the continuous accelerometer data, we characterized children into 3 groups: 1 ) 'MVA+VA' if they had $\geq 60$ min of MVA each day and $\geq 10$ min of VA on at least 3 days, 2) 'MVA only' if they had $\geq 60$ min of MVA each day but did not have $\geq 10$ min of VA on at least 3 days, and 3) 'non-active' if they had $<60$ min of MVA on any one day. In multivariable linear regression models, due to the smaller sample sizes in the 2 active groups, we combined the MVA+VA and MVA only groups, yielding a dichotomized predictor variable, 'active' versus 'non-active'.

\subsection{Personal black carbon (BC) monitoring}

Personal exposure to $\mathrm{BC}$ was measured over two 24-h periods at the beginning and end of the 6-d activity-monitoring period (Fig. 2). Children carried a MicroAeth (Model AE51, Magee

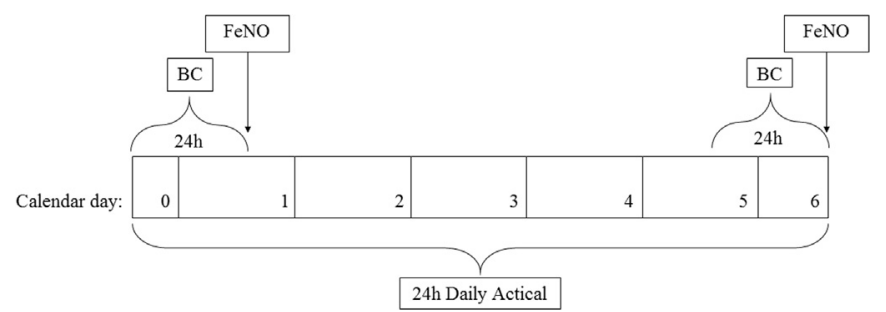

Fig. 2. Sampling scheme for actical, BC, and FeNO collection. BC: black carbon, FeNO: fractional exhaled nitric oxide. 
Scientific, Berkley, CA) inside a vest pocket that contained an air inlet in the breathing zone (vest collar) (Cai et al., 2013, 2014). Children were instructed to remove the vest during vigorous activity and to keep it nearby and uncovered. Every 5-min data were averaged to yield a mean 24-h exposure assessment. We previously have demonstrated excellent compliance with wearing the $\mathrm{BC}$ monitoring equipment in this age group (Lovinsky-Desir et al., 2014). In addition, MVA did not vary significantly between days in which the vest was worn and was not worn (Signed rank test, $\mathrm{p}=0.40$ ).

BC data were cleaned according to algorithms developed to account for false positive and negative data that can result from physical vibration even though they were shown to not impact long term ( $\geq 2$ h) averages (Cai et al., 2013, 2014). Cleaned 5 min data were then averaged to yield a mean 24-h exposure assessment. Mean 24-h personal exposure to BC moderately correlated across the two days of measurement (Pearson $r=0.36, p<0.01$ ), reflecting some day-to-day variation in exposure. 50\% of the personal BC exposure measurements were sampled during the NYC cold weather-heating season (ie October-April).

\subsection{Fractional exhaled nitric oxide (FeNO) measurement}

FeNO was collected twice during the sampling week, immediately following both 24-h BC collection periods, using the offline method (GE Instruments, Boulder, CO) (Fig. 2) (Perzanowski et al., 2008). Participants refrained from physical activity for at least $1 \mathrm{~h}$ prior to sample collection. Three breath samples were collected in individual Mylar balloons at a flow rate of $83 \mathrm{~mL} / \mathrm{s}$ and results were averaged to give a mean FeNO for each of the two sampling days. For subjects with only one FeNO measurement $(n=16)$ due to equipment failure, or inability to collect an adequate sample, the one-day measurement was used in place of an average. In addition, to account for possible home environmental contamination of our sample, two ambient air samples were collected with a nitric oxide analyzer (GE Instruments, Boulder, CO) and averaged.

\subsection{Specific allergen sensitization assessment}

Relationships between BC exposure, FeNO, and asthma outcomes have been shown to differ among allergic and non-allergic children (Cornell et al., 2012; Jung et al., 2012). Therefore, we tested allergic sensitization to indoor and outdoor allergens commonly found in urban environments (German cockroach, mouse urine proteins, dust mites (Dermatophagoides farina), common ragweed, mixed grass pollen (Gx2), and mixed tree pollen (Tx1)). Serum samples were collected at ages 7, 9 and 11. Age 9 or age 7 data were used for the children that did not have a sample available at age 11. Specific immunoglobulin (Ig)E was measured using Immunocap (Phadia, Uppsala, Sweden) (Donohue et al., 2008). Common ragweed, mixed grass pollen and mixed tree pollen data were not available for $n=13$ children that were included in this study. Allergen-specific IgE levels of $0.35 \mathrm{IU} / \mathrm{mL}$ or greater were considered positive.

\subsection{Statistical analysis}

Chi-square tests were used to determine differences among 3 mutually exclusive groups of activity (ie. MVA+VA, MVA only, and non-active) by sex, asthma diagnosis, and obesity. For each child, the two samples of 24-h personal BC and FeNO were averaged and $\log$ transformed to assume normal distribution. Student's $t$-tests were used to determine differences in personal BC and FeNO by physical activity group.

In multivariable linear regression models, we combined the
MVA+VA and MVA only groups, yielding a dichotomized predictor variable, 'active' versus 'non-active.' The outcomes of interest were personal BC and FeNO. Using step-wise linear regression, models were adjusted for covariates including age, sex, race/ ethnicity, BMI percentile, second hand smoke (SHS) exposure within the last 2 weeks (determined by questionnaire), season of measurement (heating vs. non-heating), allergen sensitization and ambient NO for FeNO models. In addition to age, sex, race/ethnicity, and standardized BMI z-score, only significant covariates were included in final models (ambient NO, cockroach sensitization and total IgE for FeNO models). We performed sensitivity analysis to assess if number of days with $\geq 60$ min of MVA was associated with personal $\mathrm{BC}$ exposure measurements. Also, we performed sensitivity analysis to account for potential environmental contamination of NO in our FeNO models. To determine the influence of personal $\mathrm{BC}$ on the relationship between activity and FeNO we stratified our analysis by $\mathrm{BC}$ concentrations at the upper tertile $\left(\geq 1785 \mathrm{ng} / \mathrm{m}^{3}\right.$ ) and examined relationships in subgroups. In addition, we performed secondary stratified analyses to explore relationships by subgroups of interests (school grade level, season of measurement, cockroach sensitization, and asthma diagnosis) for future hypothesis generation.

A significance level of 0.05 was established a priori. All statistical analyses were performed using SAS 9.4.

\section{Results}

\subsection{Subject characteristics}

Demographic characteristics of the 129 children in our cohort are included in Table 1 . The children were generally active with $55.8 \%$ meeting the CDC recommendation of $\geq 60 \mathrm{~min}$ of MVA daily (Fig. S1). Among the active group, only 20 children met the additional criteria of $\geq 10 \mathrm{~min}$ of vigorous activity on at least 3 days (MVA+VA). Activity patterns significantly varied by sex $(\mathrm{p}<0.01)$ with more boys meeting the additional VA criteria than girls (Fig. S2). Activity patterns did not vary by current asthma $(\mathrm{p}=0.77)$ or by obesity $(\mathrm{p}=0.81$, Fig. $\mathrm{S} 2)$.

\subsection{Physical activity was associated with greater personal $B C$ exposure}

There was a trend towards higher average personal $\mathrm{BC}$ concentrations among the active children ( $n=72, \mathrm{GM} \pm \mathrm{SD}$ : $\left.1210 \pm 1.78 \mathrm{ng} / \mathrm{m}^{3}\right)$ compared to the non-active children $(\mathrm{n}=57$, $\left.\mathrm{GM} \pm \mathrm{SD}: 1060 \pm 1.69 \mathrm{ng} / \mathrm{m}^{3}\right)(\mathrm{p}=0.11)$. Specifically, the active children that met the additional VA criteria had significantly greater personal $B C$ concentrations $(n=20, \quad G M \pm S D$ : $\left.1400 \pm 1.65 \mathrm{ng} / \mathrm{m}^{3}\right)$ compared to the non-active children $(\mathrm{p}=0.04)$ (Fig. 3, Table S2). In multivariable linear regression models, after controlling for age, sex, race/ethnicity, and BMI z-score, active children had $25 \%$ greater BC concentrations compared to non-active children (95\% CI: 4-51\%, $\mathrm{p}=0.02$ ) (Table 2).

In a sensitivity analysis, we reran our model using numbers of days with $\geq 60$ min of MVA as the main predictor of interest and again observed a significant positive relationship between physical activity and personal BC measures (crude $\beta$ estimate $=0.07,95 \% \mathrm{CI}$ : $0,0.14, \mathrm{p}=0.05$, adjusted $\beta$ estimate $=0.09,95 \% \mathrm{CI}: 0.02,0.15$, $\mathrm{p}=0.01$ ) (Fig. S3).

\subsection{FeNO was reduced among active children}

In bivariate analysis there was no significant difference in FeNO in the active children $(n=72, G M \pm S D$ : $14.66 \pm 2.17 \mathrm{ppb})$ compared to the non-active children $(n=57, G M \pm S D$ : $17.63 \pm 2.15$ 
Table 1

Demographic characteristics of participants in both active and non-active groups.

\begin{tabular}{llll}
\hline & $\begin{array}{l}\text { Non-active } \\
(\mathrm{n}=57)\end{array}$ & $\begin{array}{l}\text { Active } \\
(\mathrm{n}=72)\end{array}$ & p Value $^{\mathrm{a}}$ \\
\hline Age in years, median (range) & $12.7(10.5-14.0)$ & $\begin{array}{l}12.3(9.2 \\
-14.3)\end{array}$ & 0.05 \\
& $32(56 \%)$ & $33(46 \%)$ & 0.25 \\
Males, n (\%) & & $43(60 \%)$ & 0.15 \\
Race/ethnicity, n (\%) & $41(72 \%)$ & $29(40 \%)$ & \\
Hispanic & $16(28 \%)$ & $41(57 \%)$ & 0.49 \\
African American & $29(51 \%)$ & $15(21 \%)$ & 0.98 \\
Asthmab, n (\%) & $12(21 \%)$ & $39(54 \%)$ & 0.71 \\
Maternal asthma, n (\%) & $29(51 \%)$ & $13(18 \%)$ & $\mathbf{0 . 0 3}$ \\
Maternal high school degree or & & $97.5(4.6)$ & 0.44 \\
$\quad$ greater, n (\%) & $3(5 \%)$ & $19(26 \%)$ & 0.64 \\
SHS exposure ${ }^{\mathrm{c}}, \mathrm{n}(\%)$ & $79.6(4.2)$ & $1210(1.8)$ & 0.16 \\
Total IgE, GM (SD) & $13(23 \%)$ & $14.7(2.2)$ & 0.18 \\
BMI $\geq$ 95th percentile, n (\%) & $1060(1.7)$ & & \\
Black Carbon, GM (SD) & $17.6(2.2)$ & & \\
FeNO, GM (SD) & & & \\
\hline
\end{tabular}

SHS: second hand smoke, IgE: Immunoglobulin E, GM: geometric mean, SD: standard deviation, BMI: body mass index.

a Chi-square test for categorical variables and $t$-test for continuous variables.

${ }^{\mathrm{b}}$ Asthma diagnosis determined by a physician at age $5-12$ year using a priori standardized criteria. ${ }^{41}$

c Second hand smoke exposure determined by responding yes to the question "In the last 2 weeks have you been exposed to tobacco smoke in the home."

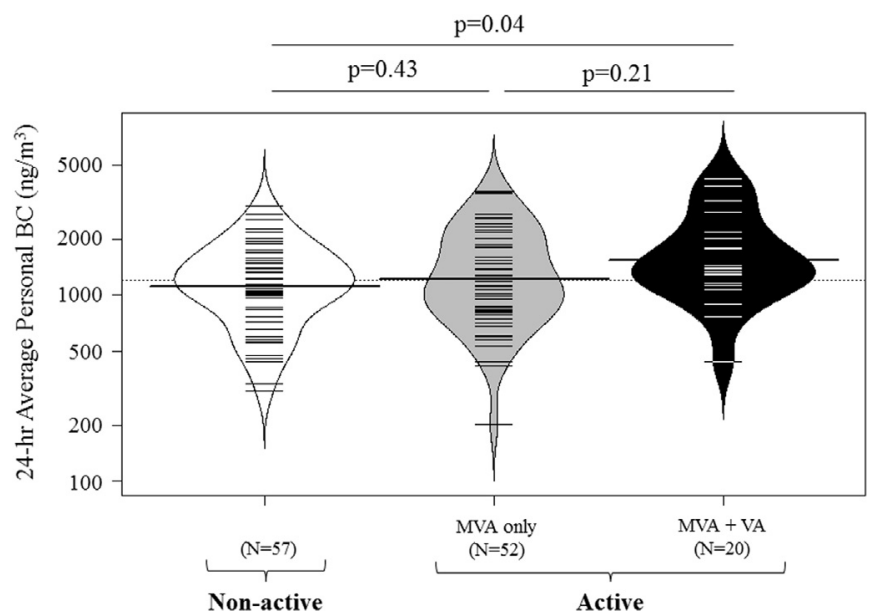

Fig. 3. Personal $B C$ exposure stratified by $C D C$ recommended physical activity levels 24-h BC exposure was averaged across 2 days of measurement and plotted on logarithmic scale. $\mathrm{N}=57$ with no daily physical activity, $\mathrm{n}=52$ with $\geq 60$ min of MVA daily, $\mathrm{n}=20$ with $\geq 60$ min MVA daily and $\geq 10$ min VA, 3 days/week. The small lines indicate individual observations, while the white, grey, and black areas demonstrate the distribution. The dotted line indicates the overall geometric mean and the thicker solid lines represent the geometric mean concentration for each group of activity. BC: black carbon, MVA: moderate to vigorous activity, VA: vigorous activity. ppb, $\mathrm{p}=0.18$ ). However, in multivariable analysis, after controlling for age, sex, race/ethnicity, BMI, cockroach sensitization, and total IgE, active children had 20\% lower FeNO than non-active children (95\% CI: $1-35 \%, p=0.04$ ) (Table 2). Sensitization to cockroach, but not to other indoor and outdoor allergens, was a significant confounder in the association between physical activity and FeNO.

In a sensitivity analysis, we removed all FeNO measurements in which the ambient NO was greater than $100 \mathrm{ppb}$ because of the possibility of home environmental contamination, yielding a sample size of $\mathrm{n}=122$. We again observed active children $(\mathrm{n}=70)$ had 21\% lower FeNO than non-active children $(n=52,95 \%$ CI: $1-$ $38 \%, \mathrm{p}=0.04)$.

\section{4. $B C$ exposure influences the relationship between activity and $\mathrm{FeNO}$}

To test the hypothesis that personal BC exposure would attenuate the relationship between activity and FeNO we stratified our linear regression model by the upper tertile of personal BC measure ( $\geq 1790 \mathrm{ng} / \mathrm{m}^{3}$ ). Among the $\mathrm{n}=33$ children that had the highest personal BC there was no association between activity and FeNO ( $\beta$ estimate $=0.00,95 \% \mathrm{CI}:-0.62,0.62, \mathrm{p}=1.00$ ). Thus, the relationship between activity and FeNO was completely driven by the $\mathrm{n}=96$ children with lower personal BC measures ( $\beta$ estimate $=-0.27,95 \% \mathrm{CI}:-0.02,-0.52, \mathrm{p}=0.04)$. Active children with lower concentrations of personal BC had 24\% lower FeNO compared to non-active children with lower BC (95\% CI: 2-41\%). However, in a formal test for interaction between activity and dichotomized $\mathrm{BC}$, the p-value for the interaction term was not significant $(\mathrm{p}=0.56)$.

\subsection{Secondary analyses}

Based on the observation that age was a significant covariate in the relationship between physical activity and $\mathrm{BC}$ measures, we stratified our linear regression model by school grade level and compared middle and high school students (grades $6-9, \mathrm{n}=91$ ) with elementary school students (grades $3-5, n=26$ ) (current grade missing for $\mathrm{n}=12$ children). Among middle and high schoolers, we detected 33\% greater BC measures in active compared to non-active children (95\% CI: 9-63\%, p < 0.01). There was a smaller and not statistically significant difference (30\%) among active versus non-active elementary school students $(95 \% \mathrm{CI}$ : $-30-141 \%, p=0.39)$. In a test for interaction the $p$-value for the interaction term was not significant $(p=0.86)$ (Table S3). BC concentrations are known to vary by heating season (Jung et al., 2010), therefore we stratified our linear regression model by NYC cold weather, heating season (ie October-April) versus non-heating season. Interestingly, among the $\mathrm{n}=64$ children that were sampled during the heating season, we identified 50\% greater BC measures in active compared to non-active children (95\% CI: $10-$

Table 2

Unadjusted and adjusted linear regression models demonstrating the effect of physical activity on FeNO and BC exposure.

\begin{tabular}{|c|c|c|c|c|c|c|}
\hline & \multicolumn{3}{|l|}{ Unadjusted Model } & \multicolumn{3}{|l|}{ Adjusted Model } \\
\hline & Parameter estimate & 95\% Confidence interval & $\mathrm{R}^{2}$ & Parameter estimate & 95\% Confidence interval & $\mathrm{R}^{2}$ \\
\hline Black carbon & 0.14 & {$[-0.06,0.33]$} & 0.02 & 0.23 & {$[0.04,0.41]^{\mathrm{a}^{*}}$} & 0.18 \\
\hline $\mathrm{FeNO}_{\text {overall }}$ & -0.19 & {$[-0.46,0.09]$} & 0.01 & -0.22 & {$[-0.43,-0.01]^{\mathrm{b}^{*}}$} & 0.49 \\
\hline $\mathrm{FeNO}_{\text {high BC }}$ & - & - & - & 0.00 & {$[-0.62,0.62]^{b}$} & 0.39 \\
\hline $\mathrm{FeNO}_{\text {lower BC }}$ & - & - & - & -0.27 & {$[-0.02,-0.53]^{\mathrm{b}^{*}}$} & 0.52 \\
\hline
\end{tabular}

High BC: $\geq 1790 \mathrm{ng} / \mathrm{m}^{3}$ (upper tertile); Lower BC: < $1790 \mathrm{ng} / \mathrm{m}^{3}$; BC: black carbon; FeNO: fractional exhaled nitric oxide.

a Model adjusted for age, sex, race/ethnicity, and BMI z-score.

${ }^{\mathrm{b}}$ Model adjusted for ambient nitric oxide, age, sex, race/ethnicity, BMI z-score, cockroach sensitization, and total IgE. p-value $<0.05$. 
$103 \%, p=0.01)$. However, the association was not statistically significant among the $\mathrm{n}=65$ children that were sampled in warm weather, non-heating season (11\% difference, $95 \% \mathrm{CI}$ : $-12-40 \%$, $\mathrm{p}=0.37$ ). In a test for interaction the $\mathrm{p}$-value for the interaction term was not significant $(\mathrm{p}=0.13)$ (Table S3).

Also, given our observation that cockroach sensitization was a significant covariate in the relationship between physical activity and FeNO we stratified our multivariable regression model by cockroach sensitization. We observed 24\% lower FeNO between the active versus non-active children that were not cockroach sensitized ( $\mathrm{n}=78,95 \% \mathrm{CI}: 3-41 \%, \mathrm{p}=0.03)$; however, among the children that were cockroach sensitized, there was no significant difference $(n=51,1 \%$ difference, $95 \% \mathrm{CI}:-35-50 \%, p=0.96)$. In a test for interaction the p-value for the interaction term was not significant $(\mathrm{p}=0.34)$ (Table S3). Similarly, when our model was stratified by asthma there was $29 \%$ lower FeNO among active versus non-active children that did not have asthma $(n=59,95 \%$ CI: $4-47 \%, p=0.02$ ). The association between physical activity and FeNO among children with asthma was not significant $(n=70,14 \%$ difference, 95\% CI: $-37-17 \%, \mathrm{p}=0.33$ ). In the test for interaction the $p$-value for the interaction was not significant $(\mathrm{p}=0.59)$ (Table S3).

\section{Discussion}

In our cohort of African American and Dominican children living in urban NYC, we found that 9-14 year olds that engaged in at least $1 \mathrm{~h}$ of physical activity daily had greater exposure to the pollutant BC. Furthermore, daily physical activity was associated with reduced airway inflammation, but primarily among the children that had lower measures of personal BC. Thus, we believe that high personal $\mathrm{BC}$ exposure offsets the protective effects of physical activity on airway inflammation.

The first evidence to suggest that physical activity among children living in polluted neighborhoods might cause asthma was published by McConnell and colleagues. They found that children living in high ozone neighborhoods in Southern California and participated in 3 or more team sports (not limited to outdoors) had an increased relative risk of developing asthma compared to children that did not play sports. This association was not detected in the low ozone neighborhoods within the same region (McConnell et al., 2002). Both ozone and BC are known to significantly impact health and climate; however, unlike ozone, to date BC has been neglected in studies of physical activity-related exposures. Some studies have suggested that increased respiratory rates and tidal volumes during vigorous activity may lead to increased deposition of pollutant particles within the lung, which could lead to asthma (Giles and Koehle, 2014; Strak et al., 2010). Also studies in adults have demonstrated that acute exposure to TRAP during various levels of physical activity can lead to acute reduction in lung function (McCreanor et al., 2007; Rundell et al., 2008). Among adolescents chronically exposed to high levels of particulate matter $(\mathrm{PM})_{2.5}$ and elemental carbon (EC) (similar component to $\mathrm{BC}$ but measured differently), Gauderman et al. (2004) demonstrated significant reduction in lung growth over an 8-year span.

One explanation for our observation that greater BC exposure occurred among those with daily physical activity is that opportunities for physical activity in urban areas may be more limited to sites of greater TRAP exposure. Time spent outdoors (Sallis et al., 1993) and access to outdoor space (Cohen et al., 2006) is associated with greater physical activity in children. Also, walking to school has been shown to have a significant contribution to daily physical activity (Cooper et al., 2010). In our urban environment, $45 \%$ of children in this study reported walking to and from school.
Outdoor TRAP exposures in urban areas can be highest along roadbeds (Jung et al., 2011). One small study of adults in NYC demonstrated that walking was associated with greater physical activity and greater $\mathrm{PM}_{2.5}$ exposure compared to traveling by car or subway (Morabia et al., 2009). In addition, we identified a significant relationship between daily physical activity and BC exposure among the older children in our cohort that could be related to increased independent outdoor activities. However, pollutant exposures are not limited to outdoor space and PM components, such as BC, can also penetrate indoors (Jung et al., 2010). $\mathrm{PM}_{2.5}$ concentrations are reportedly higher indoors than outdoors particularly in the cold weather months (Jung et al., 2010), which is also a time when children are more likely to spend time and potentially be more active indoors. And interestingly, we observed a stronger relationship between physical activity and personal BC exposure in the cold weather, heating season months compared to non-heating season months. These findings highlight the need for future studies to better identify differences between indoor and outdoor pollutant exposures during periods of physical activity.

Our finding of an inverse association between physical activity and FeNO is consistent with Mendes et al. who reported reduced FeNO among adult asthmatics that participated in a 3-month-long aerobic training program (Mendes et al., 2011). However, in two small clinical trials of children with asthma that participated in a 3-month aerobic training program there was no significant reduction in FeNO (Bonsignore et al., 2008; Moreira et al., 2008). Our study is novel because we assessed daily habitual physical activity while the previous reports assessed participation in an exercise program at least 2 days per week. Also, the above-mentioned studies focused on children with asthma, yet our sample also included children without asthma. Upon stratifying the multivariable regression models by asthma, we observed attenuation in the association between physical activity and FeNO among the children with asthma, specifically compared to healthy children. Our findings suggest that physical activity could have a protective effect among those at risk for developing airway inflammation, whereas this protection is less significant when disease has already occurred. In addition, based on our results and given the findings by Spira-Cohen et al. (2011) of a positive association between personal EC exposure and respiratory symptoms in children with asthma, we also can reason that the benefit of exercise on reducing airway inflammation, may not extend to children with asthma who are more likely to have an opposing inflammatory response linked to respiratory symptoms.

Several mechanisms to explain the relationship between exercise and airway inflammation have been explored in rodent models. For example, exercised mice have been demonstrated to have reduced expression of pro-inflammatory $\mathrm{T}$ helper (Th)-2 cytokines, ${ }^{43}$ reduced activation of the inflammatory transcription factor NF- $\kappa$ B (Pastva et al., 2004) and reduced concentrations of granulocyte colony-stimulating factor (Files et al., 2015). In addition, epigenetic changes that may occur in response to exercise and physical activity could lead to a reduction in inflammation (Horsburgh et al., 2015). However, further mechanistic studies in humans, especially in children, are needed.

We also identified cockroach sensitization was a key covariate in the relationship between physical activity and FeNO, unlike sensitization to other aeroallergens commonly found in urban environments. Combined exposure to cockroach allergen and the TRAP, polycyclic aromatic hydrocarbon (PAH) previously has been identified as an important risk factor for the development of cockroach sensitization (Jung et al., 2015; Perzanowski et al., 2013). Here we identified cockroach non-sensitized children had greater inverse associations between physical activity and FeNO compared to the sensitized children. One theory to explain resistance to activity induced reductions in airway inflammation is 
that seroatopy is such a strong predictor of FeNO (Scott et al., 2010) that associations with physical activity are blunted among the already sensitized. Similarly, in another cohort of children living in NYC, non-allergic children had significant inverse associations between BC exposure and FeNO (Cornell et al., 2012). Despite a significant difference in SHS exposure between the active and nonactive children (Table 1), SHS was not a significant covariate in the relationship between physical activity and FeNO, nor the relationship between physical activity and BC exposure.

The cross sectional design is a limitation of our study because of our lack of ability to assess temporality. Airway inflammation from allergic disease or asthma could cause urban children to reduce their physical activity; thus, we are unable to presume a causal relationship between physical activity and reduced airway inflammation. However, of the 71 asthmatic children within our study $75 \%$ answered no to the question: "Did your asthma limit your activity today?" and only 2 children reported that their asthma limited their activity most of the time. While we attempted to assess for confounding, the observational nature of the design coupled with our limited sample size makes it difficult to fully exclude unmeasured confounding. Also, the children in this cohort were of African American and Dominican descent and replication in a larger and more racially and ethnically diverse population is warranted. An additional study limitation is that our FeNO measurements were only sampled at 2 different points during the 1-week of observation and not daily. Although, we believe that averaging the repeated measures likely accounted for potential day-to-day fluctuations. Similar to our FeNO measurements, we investigated average personal exposure to BC at two 24$\mathrm{h}$ periods during a week and related that to average physical activity patterns across that same week. While this method allowed us to observe relationships between trends in exposures and behaviors, future studies are necessary to match real-time exposure and physical activity to better identify if periods of physical activity are actually occurring in locations of high exposure. In our study we did not investigate whether the activity was occurring indoors or outdoors, thus we cannot speak to where in particular the exposure was greatest. In particular future studies should address differences between indoor and outdoor activity/exposure relationships.

Exposure to many TRAPs including BC can vary greatly due to changes in traffic patterns and distance from traffic related sources (Dons et al., 2012; Patel et al., 2009). Therefore, personal exposure monitoring, as measured here, is ideal for capturing individual level BC exposure when evaluating relationships between exposures, behaviors and clinical measures. An additional strength of the study is the use of wrist mounted accelerometer devises that were not removed during the observation period. Thus, we did not rely on the children to remember to put the device on each day. Also, measurement of physical activity was not presented to the children as a primary predictor in the parent study, therefore we do not believe that participation in the study influenced physical activity levels.

\section{Conclusions}

In conclusion, using personal exposure samplers we have demonstrated that children that live in an urban environment and are physically active on a daily basis have higher personal BC exposure. High BC exposure partially offsets the protective relationship between physical activity and airway inflammation. Overall, our findings contribute to the evidence that physical activity may be beneficial to the respiratory health of children, adding further support to the current physical activity recommendations; however, high $\mathrm{BC}$ pollutant exposure attenuates this effect.

\section{Contributors' statement page}

Stephanie Lovinsky-Desir developed the concept of this particular study, performed all data analysis, drafted the initial manuscript, and approved the final manuscript as submitted.

Kyung Hwa Jung supervised data collection, advised on the analysis of the data, critically reviewed and revised the manuscript and approved of the final manuscript as submitted.

Andrew G. Rundle advised on the analysis of the data, critically reviewed the manuscript, and approved the final manuscript as submitted.

Lori A. Hoepner processed the physical activity data, critically reviewed the manuscript, and approved the final manuscript as submitted.

Joshua Bautista collected the data, critically reviewed the manuscript, and approved the final manuscript as submitted.

Frederica P. Perera established the longitudinal birth cohort, critically reviewed the manuscript, and approved the final manuscript as submitted.

Steven N. Chillrud designed and advised the black carbon data collection, critically reviewed the manuscript, and approved the final manuscript as submitted.

Matthew S. Perzanowski supervised the FeNO data collection, critically reviewed the manuscript, and approved the final manuscript as submitted.

Rachel L. Miller assisted with the original concept of the study, supervised all data collection, critically reviewed the manuscript and approved the final manuscript as submitted.

\section{Funding sources}

This work was supported by the National Institutes of Health [3R01ES013163-07S1, 2R01ES13163-06A1, 5 P01 ES09600/EPA RD83214101, P30ES009089, KL2 TR00008] and the Stony Wold Herbert Fund.

\section{Financial disclosures statement}

The authors have no financial conflicts to disclose.

\section{Conflict of interest}

The authors have no conflicts of interest to disclose.

\section{Appendix A. Supporting information}

Supplementary data associated with this article can be found in the online version at doi:10.1016/j.envres.2016.09.005.

\section{References}

Bonsignore, M.R., et al., 2008. Effects of exercise training and montelukast in children with mild asthma. Med Sci. Sports Exerc. 40, 405-412.

Cai, J., et al., 2013. Optimization approaches to ameliorate humidity and vibration related issues using the microAeth black carbon monitor for personal exposure measurement. Aerosol Sci. Technol. 47, 1196-1204.

Cai, J., et al., 2014. Validation of microaeth(R) as a black carbon monitor for fixedsite measurement and optimization for personal exposure characterization. Aerosol Air Qual. Res. 14, 1-9. 
Cohen, D.A., et al., 2006. Public parks and physical activity among adolescent girls. Pediatrics 118, e1381-e1389.

Cooper, A.R., et al., 2010. Mapping the walk to school using accelerometry combined with a global positioning system. Am. J. Prev. Med. 38, 178-183.

Cornell, A.G., et al., 2012. Domestic airborne black carbon and exhaled nitric oxide in children in NYC. J. Expo. Sci. Environ. Epidemiol. 22, 258-266.

Council on Sports, M, et al., 2006. Active healthy living: prevention of childhood obesity through increased physical activity. Pediatrics 117, 1834-1842.

Donohue, K.M., et al., 2008. Anti-cockroach and anti-mouse IgE are associated with early wheeze and atopy in an inner-city birth cohort. J. Allergy Clin. Immunol. 122, 914.

Donohue, K.M., et al., 2013. Prenatal and postnatal bisphenol A exposure and asthma development among inner-city children. J. Allergy Clin. Immunol. 131, 736-742.

Dons, E., et al., 2012. Personal exposure to Black Carbon in transport microenvironments. Atmos, Environ. 55, 392-398.

Dweik, R.A., et al., 2011. An official ATS clinical practice guideline: interpretation of exhaled nitric oxide levels (FENO) for clinical applications. Am. J. Respir. Crit. Care Med. 184, 602-615.

Files, D.C., et al., 2015. Therapeutic exercise attenuates neutrophilic lung injury and skeletal muscle wasting. Sci. Transl. Med. 7, 278 ra32.

Gauderman, W.J., et al., 2004. The effect of air pollution on lung development from 10 to 18 years of age. N. Engl. J. Med. 351, 1057-1067.

Giles, L.V., Koehle, M.S., 2014. The health effects of exercising in air pollution. Sports Med. 44, 223-249.

Horsburgh, S., et al, 2015. Exercise and inflammation-related epigenetic modifications: focus on DNA methylation. Exerc. Immunol. Rev. 21, 26-41 〈http://www. cdc.gov/physicalactivity/everyone/guidelines/children.html $>$.

Jung, K.H., et al., 2011. Seasonal gradient patterns of polycyclic aromatic hydrocarbons and particulate matter concentrations near a highway. Atmosphere 2. 533-552.

Jung, K.H., et al., 2015. Repeatedly high polycyclic aromatic hydrocarbon exposure and cockroach sensitization among inner-city children. Environ. Res. 140, 649-656.

Jung, K.H., et al., 2010. Effects of heating season on residential indoor and outdoor polycyclic aromatic hydrocarbons, black carbon, and particulate matter in an urban birth cohort. Atmos. Environ. 44, 4545-4552.

Jung, K.H., et al., 2012. Repeated exposure to polycyclic aromatic hydrocarbons and asthma: effect of seroatopy. Ann. Allergy Asthma Immunol. 109, 249-254.

Lin, W., et al., 2011. Acute respiratory inflammation in children and black carbon in ambient air before and during the 2008 Beijing Olympics. Environ. Health Perspect. 119, 1507-1512.

Lovinsky-Desir, S., et al., 2014. Urban adolescents readily comply with a complicated asthma research protocol. Clin. Med. Insights Circ. Respir. Pulm. Med. 8, 5-9.

McConnell, R., et al., 2002. Asthma in exercising children exposed to ozone: a cohort study. Lancet 359, 386-391.

McConnell, R., et al., 2006. Traffic, susceptibility, and childhood asthma. Environ. Health Perspect. 114, 766-772.

McCreanor, J., et al., 2007. Respiratory effects of exposure to diesel traffic in persons with asthma. N. Engl. J. Med. 357, 2348-2358.

Mendes, F.A., et al., 2011. Effects of aerobic training on airway inflammation in asthmatic patients. Med. Sci. Sports Exerc. 43, 197-203.

Morabia, A., et al., 2009. Air pollution and activity during transportation by car, subway, and walking. Am. J. Prev. Med. 37, 72-77.

Moreira, A., et al., 2008. Physical training does not increase allergic inflammation in asthmatic children. Eur. Respir. J. 32, 1570-1575.

Oravisjarvi, K., et al., 2011. Effects of physical activity on the deposition of trafficrelated particles into the human lungs in silico. Sci. Total Environ. 409, 4511-4518.

Pastva, A., et al., 2004. Aerobic exercise attenuates airway inflammatory responses in a mouse model of atopic asthma. J. Immunol. 172, 4520-4526.

Patel, M.M., et al., 2009. Spatial and temporal variations in traffic-related particulate matter at new york city high schools. Atmos. Environ. 43, 4975-4981.

Perera, F.P., et al., 2003. Effects of transplacental exposure to environmental pollutants on birth outcomes in a multiethnic population. Environ. Health Perspect. 111, 201-205.

Perzanowski, M.S., et al., 2013. Early-life cockroach allergen and polycyclic aromatic hydrocarbon exposures predict cockroach sensitization among inner-city children. J. Allergy Clin. Immunol. 131, 886-893.

Perzanowski, M.S., et al., 2008. Modifications improve an offline exhaled nitric oxide collection device for use with young children. J. Allergy Clin. Immunol. 122,213 , author reply 214.

Rundell, K.W., et al., 2008. Decreased lung function after inhalation of ultrafine and fine particulate matter during exercise is related to decreased total nitrate in exhaled breath condensate. Inhal. Toxicol. 20, 1-9.

Rundle, A., et al., 2009. Physical activity and asthma symptoms among New York City Head Start Children. J. Asthma 46, 803-809.

Sallis, J.F., et al., 1993. Correlates of physical activity at home in Mexican-American and Anglo-American preschool children. Health Psychol. 12, 390-398.

Scott, M., et al., 2010. Influence of atopy and asthma on exhaled nitric oxide in an unselected birth cohort study. Thorax 65, 258-262.

Spira-Cohen, A., et al., 2011. Personal exposures to traffic-related air pollution and acute respiratory health among Bronx schoolchildren with asthma. Environ. Health Perspect. 119, 559-565.

Strak, M., et al., 2010. Respiratory health effects of ultrafine and fine particle exposure in cyclists. Occup. Environ. Med. 67, 118-124.

Strong, W.B., et al., 2005. Evidence based physical activity for school-age youth. J. Pedia. 146, 732-737.

Wallack, J.S., Ramanathan, V., 2009. The other climate changers why black carbon and ozone also matter. Foreign Aff. 88, 105-113. 